\title{
Limb segment inclination sense in proprioception*
}

\author{
C. J. Worringham**, G.E. Stelmach, and Z.E. Martin \\ Motor Behavior Laboratory, University of Wisconsin, 2000 Observatory Drive, Madison, WI 53706, USA
}

Summary. Two experiments were performed to determine if proprioceptive signals are perceived more readily in terms of limb segment inclinations to the vertical than as joint angles. Subjects attempted to match arm positions with the upper arms supported at different inclinations. Constant error data showed that, when instructed to match forearm inclinations to the vertical, subjects were very accurate. When required to match elbow joint angles, however, errors were strongly biased in the direction of matching forearm inclinations. The results support a view of proprioception as a system in which afferent signals related to the gravitational torques acting at joints lead to the perception of limb orientation rather than joint angles. Such a system would allow more efficient determination of the relationship of limb segments to external objects than would one based purely on joint angles.

Key words: Joint angle sense - Inclination sense Gravitational torque - Proprioception

\section{Introduction}

A traditional view of proprioception is that it can be equated with joint angle sensation. Physiological work which documented the maximal firing of individual joint receptors at specific angular displacements (Andrew and Dodt 1953; Skoglund 1956; Kelso and Stelmach 1978) lent support to this view.

\footnotetext{
* This work was supported in part by research awards from the U.S. Public Health Service, AG05154, NS17421 and NATO no $227 / 82$

** Present address: Department of Kinesiology, 401 Washtenaw Avenue, University of Michigan, Ann Arbor, MI 48109-2214, USA

Offprint requests to: G.E. Stelmach (address see above)
}

In this paper we describe experiments which tested this assumption by comparing the accuracy with which joint angles and limb segment inclinations can be matched.

That the properties of joint afferents are not those of simple angle transducers has been apparent since the demonstration by Burgess and Clark (1969) that afferent fiber firing patterns are far from even throughout the range of motion, and that they may occur at different joint angles. Nevertheless, the implicit assumption that joint angle is the fundamental parameter in proprioception has persisted, even though other sensory systems with only indirect relationships to joint angle contribute to proprioception, most notably muscle afferents (Matthews 1977; McCloskey et al. 1983) but also cutaneous inputs (Moberg 1983).

There are good reasons for supposing that the inclination of a limb to the gravitational vertical may often be both more useful and more readily available to the central nervous system than information about joint angle per se. One consideration is that gravity provides a stable vertical reference frame relative to the array of primarily static objects which we manipulate and reach for in daily activities. Conversely, a joint angle reference frame does not have an unchanging relationship to external objects. At issue here is a recurrent theme in motor control - the relative computational complexity of the alternative systems (Stelmach and Diggles 1982). For certain purposes, there is little difference between the alternatives in terms of computation. To determine, for example, the distance between the hand and the shoulder when the arm is in the sagittal plane, a joint angle-based system would require implicit knowledge of two limb segment lengths, and of two joint angles. An inclination based system would also need to 'know' the two segment lengths and two inclinations. Of course proprioception would be of limited value if 
it allowed only the determination of body parts relative to one another rather than to external objects and surfaces (Kelso and Stelmach 1978). However, as we will show, even with internal comparisons between positions of body parts, inclination information can be predominant.

Other tasks require that the orientation of a distal segment be known: whether, for example, the hand is holding a glass of water level. Here, a system using solely joint angle inputs would have to determine the orientation of the head through vision or vestibular information (or of the feet relative to the ground), and would also require knowledge of the angles of all intervening joints between the head (or feet) and the hand. By contrast, an inclination system can dispense with all joint angle inputs, needing only the inclination of the distal limb segment, which can be provided through the relative torque at the neighboring joint (Worringham and Stelmach 1985).

The first to propose that orientation to the vertical rather than joint angles per se might subserve limb position sense was Soechting (1982), who found less variability in the matching of forearm inclinations to the vertical than in matching elbow joint angles, when upper arm inclinations differed. Soechting and Ross (1984) have subsequently provided more evidence in support of this view. What tends to diminish the strength of Soechting's conclusions is the fact that they were founded on variable error data. Greater variability in matching may allow inferences regarding the acuity of alternative reference systems, but does not distinguish between the primacy of one type of information over the other. The appropriate measure is constant error, since the hypothesized primacy of inclination-related afference gives rise to a quantitative, directional prediction for error patterns. Specifically, subjects should be biased towards the matching of limb inclinations when instructed to match joint angles - but not vice versa when the segments proximal to the joint have different inclinations. A hypothetical, perfect inclination match is shown in Fig. 1b. For comparison, a hypothetical, perfect joint angle match is depicted in Fig. 1c.

The experiments reported below were designed to test the hypothesis that subjects would have small constant errors when told to match inclinations, but that they would have large constant errors when told to match joint angles - in the direction and of a magnitude which would indicate a bias towards inclination matching. Such an outcome would provide much more direct support for the primacy of a gravitational reference frame over a joint angle reference frame than can be forthcoming from variable error data.

\section{Experiment one}

\section{Methods}

Two males and eight females, with an average age of $22.6 \pm 1.7$ years, acted as subjects. Their participation was one option of an undergraduate course requirement at the University of WisconsinMadison. They were unpaid and had no prior knowledge of the experimental hypothesis.

A self-aligning electrogoniometer (Therapeutics Unlimited ULGN-67) was attached to the lateral surface of each of the subject's arms with double-sided adhesive tape. The axis of rotation was centered over the trochlear notch of the ulna. Care was taken to position the goniometers so that no undue stretching of the skin or other position-related cues would be present. The electrogoniometers were calibrated prior to each testing session, and had a resolution of approximately half a degree. Their output was recorded by a PDP-11/73 minicomputer at discrete times by the experimenter pressing a key on a computer terminal in the testing chamber. After analog to digital conversion, the voltage input was converted to degrees of arc using regression equations automatically derived from the calibration data.

Three target angles: $15^{\circ}, 35^{\circ}$, and $55^{\circ}$ of inclination from the vertical were marked on a plexiglass plate between the subject's arms. These positions were used by the experimenter to ensure correct alignment of the subject's target arm (i.e. the arm which moved to the criterion position at the outset of each trial. The other arm was designated the matching arm).

Subjects were informed that their task would be that of matching arm positions in two different conditions "elbow joint angle matching" and "forearm inclination matching". These were explained to the subjects in the following terms: the elbow joint angle was defined as the angle between the upper arm and the forearm, and when told to match joint angles, subjects were instructed to concentrate on trying to make these angles the same for the two arms. Forearm inclination was defined as the angle which the forearm makes to an imaginary vertical line, and when told to match inclinations, subjects were required to make these inclinations the same for each arm. In addition to verbal descriptions, both types of angles were demonstrated by the experimenter. Subjects were also told that in inclination matching, the two forearms should be parallel to one another. All subjects were asked whether they fully understood the difference between these two types of angles before preceeding, and further clarification was given as necessary.

After the attachment of the electrogoniometers, subjects were blindfolded and seated in a chair at a table. Their upper arms were positioned on two support surfaces whose inclinations differed by $20^{\circ}$, so that the target arm and matching arm elbow angles were $165^{\circ}$ and $145^{\circ}$ when the forearms were in a horizontal resting position, denoted below as the reference position. The arms were positioned so that they moved in parasagittal planes. This set-up is illustrated in Fig. 1a.

Since the placement of each electrogoniometer was likely to differ slightly between the two arms, the subject's forearms were rested in the reference position shown in Fig. 1a, and the angles were then sampled. Any differences between the recorded angle and the defined angles of $165^{\circ}$ and $145^{\circ}$ were then used to determine an offset for each electrogoniometer signal to correct for any error in their placement. This reference position was sampled at the beginning of testing and again every seven trials, so that mean offsets representative of the whole sessions were used. All experimental trials began, however, with the target forearm resting on a removable support surface which inclined it about $10^{\circ}$ steeper than the matching arm. This manipulation rendered angular displacement information unreliable and its effect neutral 


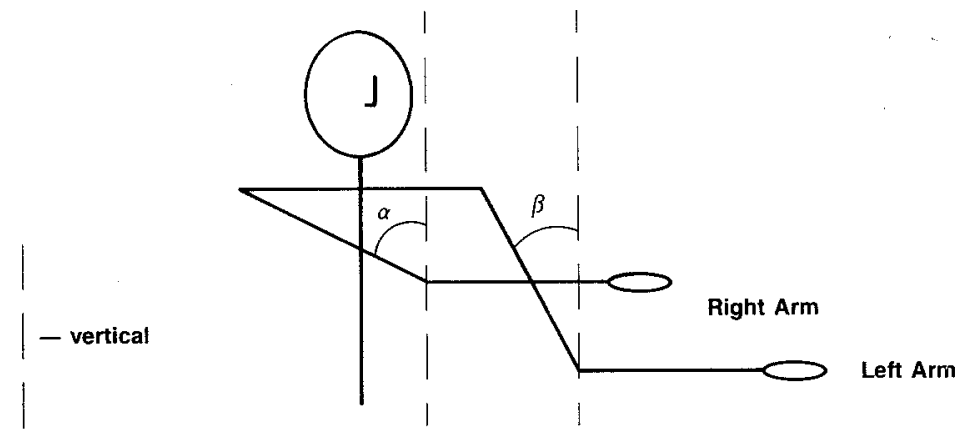

Fig. 1a. Inclination of upper arms differed by $20^{\circ}$ throughout both experiments. $\left(\alpha=75^{\circ}, \beta=55^{\circ}\right)$

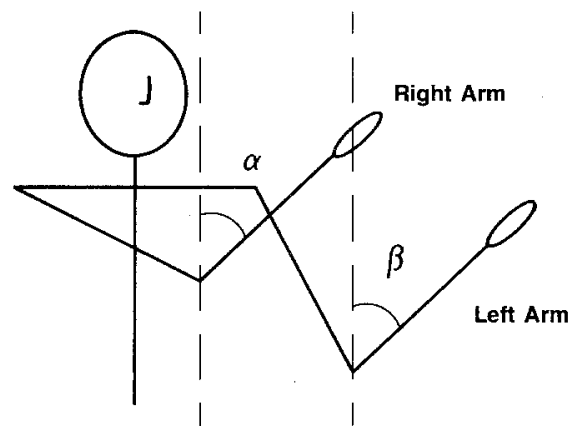

Fig. 1b. Hypothetical final position with forearm inclinations perfectly matched.

$(\alpha=\beta)$

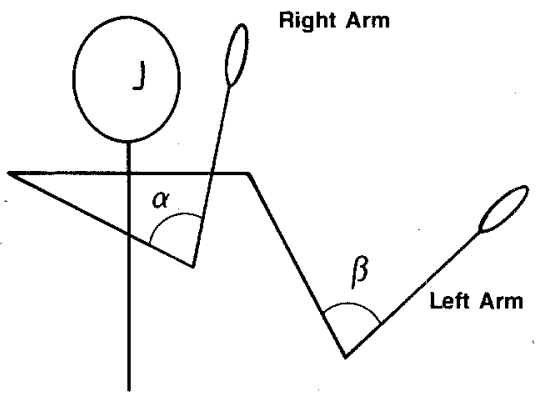

Fig. 1c. Hypothetical final position with elbow angles perfectly matched. $(\alpha=\beta)$

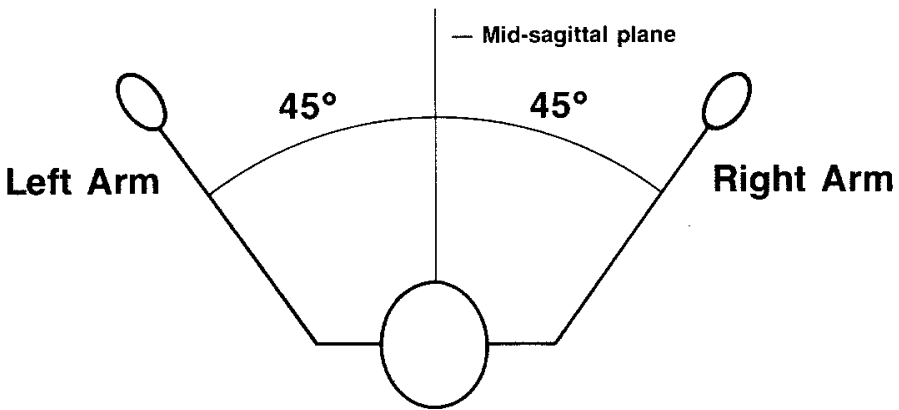

Fig. 1d. Overhead view of left and right arm positions in Experiment Two.

Arm movements took place in the vertical plane.

Fig. 1. Arm positions for Experiments one and two, and for hypothetical inclination and joint angle matching 
for the two conditions, since a subject who moved the matching arm through the same angle as the target arm would have errors of about $10^{\circ}$, midway between the positions representing perfect matching in the two conditions (see Figs. 1b, c).

At the beginning of each trial, subjects were told to slowly flex the elbow of the target arm until to stop (the criterion positions were thus experimenter-determined, not pre-selected). The experimenter told the subject to stop when close to one of the three target angles. The command "joint angle - match" or "inclination - match" was then given, and the subject matched the appropriate angles at his or her own speed, without restrictions on fine adjustments at the end of the movement. The subject said "now" when satisfied that the match had been made, and the positions were then sampled. The subject was then told to lower one arm, and then the other, to the starting positions, ready for the next trial. Halfway through the experiment, the supports for the subject's upper arms were repositioned so that the opposite limb served as the target arm.

The two experimental conditions of joint angle and inclination matching were fully crossed with the three target angles, and with each arm serving as the target limb. There were eight repetitions of each combination, making a total of 96 data trials per subject. In addition, 16 'reference position' trials were used to calculate offsets as explained above. Equal numbers of subjects began with the left arm and right arm serving as the target limb. The order of conditions was random, with the restriction that no combination of angle and type of match could occur on two consecutive trials.

For joint angle matching, the measured joint angles were used directly, after misalignment offsets were accounted for. For inclination matching, an additional $20^{\circ}$ offset was used to account for the differing upper arm inclinations, and $55^{\circ}$ were subtracted from each joint angle to express the forearm positions as inclinations to the vertical. Constant error, the algebraic deviations from a perfect target angle match, and variable error, the standard deviation of the algebraic deviations of target angles were recorded and analyzed (Stelmach 1973). Positive and negative CEs indicated that the matching limb respectively overshot or undershot the position which would satisfy the relevant type of match. For joint angle matching, therefore, positive CEs indicate a bias towards inclination matching, while for inclination matching negative CEs reflect a tendency towards joint angle matching. Data were analyzed with a 3-factor repeated measures analysis of variance involving 2 conditions, 3 target angles, and the 2 arms, each of which served as target arm.

\section{Results}

The group mean constant errors for the six combinations of condition and joint angle are shown in Fig. 2. As predicted, forearm inclination matching was performed very accurately, with a mean $\mathrm{CE}$ of $-1.16^{\circ}$. The elbow joint angle matching condition had a large positive $\mathrm{CE}$ averaging $17.1^{\circ}$. The main effect of condition was statistically significant: $\mathrm{F} 1,9=618.5$, $p<0.001$. The direction and magnitude of the CE for joint angle matching shows that subjects were strongly biased towards the achievement of positions in which the forearm inclinations were matched, but there was only minimal bias in the opposite direction during inclination matching trials. This effect was present at all three target angles and for both arms. The only other significant effect was for target angle,

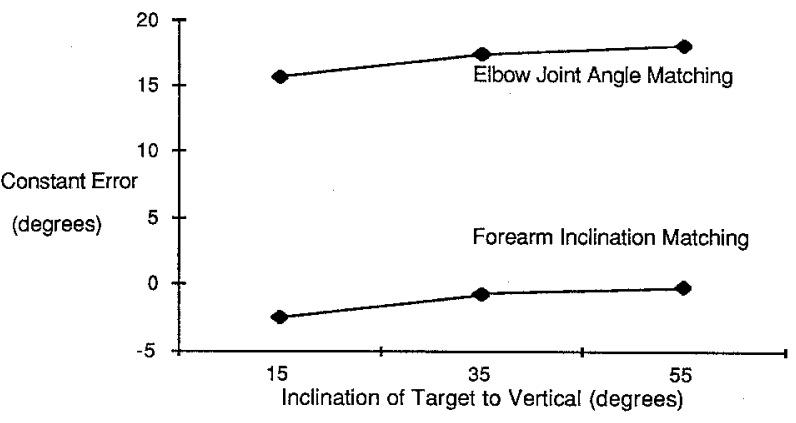

Fig. 2. Constant error for Experiment one

Table 1. Mean variable error for conditions and target angles

\begin{tabular}{llll}
\hline Target angle: & $15^{\circ}$ & $35^{\circ}$ & $55^{\circ}$ \\
\hline Experiment one & & & \\
$\quad$ Target arm: left & & & \\
$\quad$ Joint angle matching: & $6.3^{\circ}$ & $4.9^{\circ}$ & $5.2^{\circ}$ \\
$\quad$ Inclination matching: & $6.5^{\circ}$ & $3.4^{\circ}$ & $4.1^{\circ}$ \\
$\quad$ Target arm: right & & & \\
$\quad$ Joint angle matching: & $3.9^{\circ}$ & $5.2^{\circ}$ & $4.6^{\circ}$ \\
$\quad$ Inclination matching: & $3.8^{\circ}$ & $4.0^{\circ}$ & $4.2^{\circ}$ \\
\hline Experiment two & & & \\
Target arm: left & & & \\
Joint angle matching: & $4.5^{\circ}$ & $5.3^{\circ}$ & $5.2^{\circ}$ \\
Inclination matching: & $5.1^{\circ}$ & $5.9^{\circ}$ & $5.5^{\circ}$ \\
Target arm: right & & & \\
Joint angle matching: & $4.6^{\circ}$ & $5.3^{\circ}$ & $4.9^{\circ}$ \\
Inclination matching: & $3.6^{\circ}$ & $4.7^{\circ}$ & $4.4^{\circ}$ \\
\hline
\end{tabular}

such that subjects were slightly closer to perfect inclination matching at the angles closer to the horizontal, F2, $18=5.7, p<0.05$, for both types of matching.

The only significant effect for variable error was an interaction between arm and target angle: F2, $18=10.2, p<0.001$. At the angle closest to vertical, $15^{\circ}$, the left arm had less variable error. VE scores are shown in Table 1 , and span less than $2.5^{\circ}$.

\section{Discussion}

The results conformed to the experiment's hypothesis and are considered in the General Discussion together with those for Experiment two. Two factors may conceivably have caused subjects to attempt inclination matching in both conditions in Experiment one, thus leading to the striking superiority of inclination matching. The instructions given subjects were not exactly equivalent for the two types of matching, since they were fold that the forearms should be made parallel in inclination 


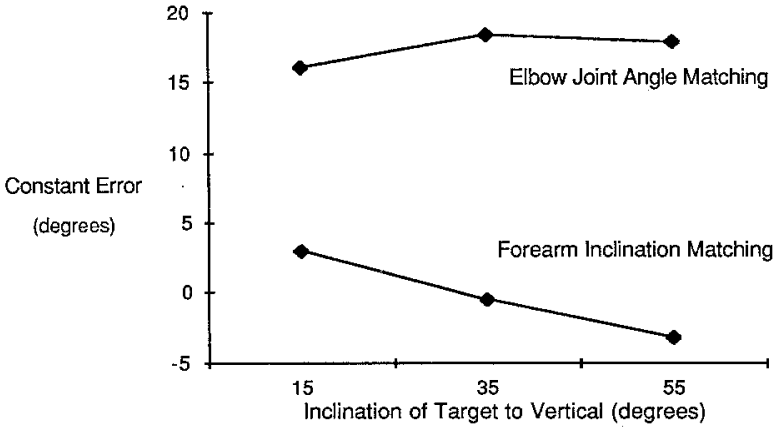

Fig. 3. Constant error for Experiment two

matching. Moreover, the fact that the two forearms were moved in parallel sagittal planes may have made it easier for subjects to "visualize" equivalent, parallel inclination angles for the two limbs. It was, therefore, decided to repeat the experiment using strictly equivalent definitions of the two types of matching, without any mention of making the arms parallel. It was furhter decided to have the arms move in perpendicular planes, to make a strategy of visualizing the arms as parallel more difficult.

\section{Experiment two}

\section{Methods}

An additional 10 subjects were recruited in the same manner as for Experiment one. There were 9 females and 1 male, and their average age was $21.5 \pm 0.9$ years. The apparatus was the same as that used in Experiment one, as were the procedure and design in all but two respects. To establish whether similar results would obtain when arm movements were not both in parallel parasagittal planes, the left and right arms were positioned at $45^{\circ}$ to left and right of the mid-sagittal plane, respectively, as shown in Fig. 1d.

A modification was made to the instructions given to subjects. The definitions of elbow joint angle and forearm inclination matching were given as in Experiment one, but the additional observation that the forearms would be parallel in inclination matching was not given.

\section{Results}

Experiment two had an outcome very similar to that of Experiment one. The group mean $\mathrm{CE}$ for joint angle matching was $17.4^{\circ}$, while that for inclination matching was $-0.2^{\circ}$. This difference was statistically significant: $\mathrm{Fl}, 9=345.2, p<0.001$. The CEs for different angle and condition combinations are shown in Fig. 3. There were no other significant main or interaction effects for constant error.

An interaction between arm and condition was present for variable error, $\mathrm{F} 1,9=6.2, p<0.05$, but no other effects were found. Group means for the twelve condition-target angle-arm combinations spanned less than one degree, as can be seen in Table 1.

\section{General discussion}

The principal result of both experiments is that the judgement of joint angles was both inaccurate and biased, in contrast to judgements about inclination. Subjects had a strong tendency to match forearm inclinations even in the joint angle matching trails, despite the clear definition of elbow joint angle, and the explicit instructions to match only the elbow joint angles in this condition. A proprioceptive system based on joint angles would have little utility if it is so readily disrupted by changing limb inclinations, in other words, if it cannot function as a fully independent and stable frame of reference. Our data confirm the speculations of Soechting (1982), but do not accord with his findings for variable error ${ }^{1}$. Indeed, our VE data alone fail to differentiate between performance in the two conditions, whereas CE does so very clearly. We are unsure why variability was not affected similarly by the conditions used in the two studies.

Some observations concerning differences in the two experiments are warranted. The purpose of the modified procedure and instructions in Experiment two was to exclude possible strategies which might differentially affect performance in the two conditions (Stelmach and Hughes 1983). In addition to the definitions of joint angles and inclinations, subjects in Experiment one were told that in inclination matching, the two forearms should be made parallel. While it is not clear that this observation would benefit subjects, additional emphasis on this condition could conceivably have led them to attempt inclination matching in joint angle matching trials, despite instructions. In Experiment two the definitions of conditions were given equal emphasis. The repositioning of the planes of motion from co-planar to orthogonal diminished the likelihood that subjects would consider trying to make the arms parallel. It also made it more difficult for subjects to visualize the relative inclinations of the arms since they were no longer in a position which would permit both to be viewed simultaneously, had vision been permitted. The absence of differences in the effects of type of matching between Experiments one and two leads us

\footnotetext{
1 This difference could be the result of different calculation methods. Soechting's method relys on the mean square difference between the error data and perfect performance
} 
to believe that subjects had tried to perform the matching as originally intended.

Comparison of Fig. 2 and 3 and inspection of Table 1 reveals minor differences in the results. We are uncertain as to why VE was slightly smaller when the left arm matched the right than vice versa for joint angle matching but not for inclination matching in Experiment two, and attribute no special importance to this outcome. Equally, we offer no explanation for the slight tendency of the left arm to match the right with less variability at the steepest target angle in Experiment one.

When the arms in separate planes, there was a tendency for the CEs to converge at the target angle closest to the vertical. While the interaction of condition and target angle was not significant, this tendency in Experiment two's data could be accounted for if inclinations are harder to judge when they approach the vertical. Since joint torques decrease at steeper inclinations, this trend is compatible with the mechanism of torque usage which we believe may underlie the sensing of inclinations. It is not clear, however, why this interaction was absent in Experiment one.

Overall, the data support the hypothesis that limb positions are sensed more naturally in terms of their inclinations than in terms of the angles at neighboring joints. We maintain, as stated elsewhere (Worringham and Stelmach 1983), that gravitational torques acting at joints may provide the relevant input for the perception of inclinations, although the manner in which the various afferent signals give rise to inclination sensation is not clear. We accept that some of these signals do indeed code joint angles, but muscle length and tension and joint reaction force signals are also present: inputs which are more closely related to gravitational torques - at least in static equilibrium and slow movement. Other sensations not normally considered proprioceptive are also inclination dependent, such as circulatory changes in a limb brought about by varying orientations in the gravitational field. Whatever the nature of the 'raw' proprioceptive signals, it seems that by the time they become available to conscious perception, they are interpreted quite readily in terms of limb segment inclination.

\section{References}

Andrew BL, Dodt E (1953) The deployment of sensory nerve endings at the knee joint of the cat. Acta Physiol Scand 28: 287-296

Burgess PR, Clark FJ (1969) Characteristics of knee joint receptors in the cat. J Physiol (Lond) 203: 317-335

Kelso JAS, Stelmach GE (1978) Central and peripheral mechanisms in motor control. In: Stelmach GE (ed) Motor control: issues and trends. New York, Academic Press, pp 1-35

Matthews PBC (1977) Muscle afferents and kinesthesia. Br Med Bull 33: 137-142

McCloskey DI, Cross MJ, Honner R, Potter EK (1983) Sensory effects of pulling and vibrating exposed tendons in man. Brain 106: $21-37$

Moberg E (1983) The role of cutaneous afferents in position sense, kinaesthesia, and motor function of the hand. Brain 106: 1-19

Soechting JF (1982) Does position sense at the elbow reflect a sense of elbow joint angle or one of limb orientation? Brain Res 248: 392-395

Soechting JF, Ross B (1984) Psychophysical determination of coordinate representation of human arm orientation. Neuroscience 13: 595-604

Skoglund S (1956) Anatomical and physiological studies of knee joint innervation in the cat. Acta Physiol Scand 36: Suppl 124: $1-101$

Stelmach GE (1973) Feedback: a determiner of forgetting in STMM. Acta Psychol 37: 333-339

Stelmach GE, Diggles VA (1982) Control theories in motor behavior. Acta Psychol 50: 83-105

Stelmach GE, Hughes BG (1983) Cognitivism and a theory of action. In: Prinz W, Sanders A (eds) Cognition and motor processes. Springer, Heidelberg New York Tokyo, pp 3-18

Worringham CJ, Stelmach GE (1985) The contribution of gravitational torques to limb position sense. Exp Brain Res 61: 38 42

Received September 26, 1986 / Accepted December 31, 1986 